\title{
Production of clusters and thin films of nitrides, oxides and carbides by pulsed laser ablation and deposition
}

\author{
S. Orlando, ${ }^{1}$ A. Paladini, ${ }^{2}$ A. Santagata, ${ }^{1}$ V. Marotta, ${ }^{1}$ G. P. Parisi, ${ }^{1}$ M. Satta, ${ }^{1}$ \\ D. Scuderi, ${ }^{2,3}$ D. Catone, ${ }^{2}$ A. Giardini, ${ }^{2}$ and A. Mele ${ }^{2, \dagger}$ \\ ${ }^{1}$ CNR-IMIP (sezione Istituto Materiali Speciali), I-85050 Tito Scalo (Pz), Italy \\ 2 Dipartimento di Chimica, Università di Roma "La Sapienza", pl. A. Moro 5, I-00185 Roma, Italy \\ ${ }^{3}$ Facoltà di Farmacia, Dipartimento di Studi di Chimica e Tecnologia delle Sostanze Biologicamente Attive, \\ Università di Roma "La Sapienza", pl. A. Moro 5, I-00185 Roma, Italy
}

\begin{abstract}
A short introduction on the principles of laser-matter interaction, material evaporation, plume formation, its reactivity with suitable gases and finally deposition are here illustrated. Experiments by mass spectrometry of formation of clusters, precursors of thin films and nanoparticles of oxides, nitrides and carbides by pulsed laser ablation (PLA) are reported. Pulsed laser ablation of targets combined with an intense atomic source produced by radiofrequency (RF), are discussed in terms of generating chemical reactions or supplying the loss of volatile components.
\end{abstract}

\section{INTRODUCTION}

Nanoparticles and thin films of various materials such as metals, semi and superconductors, nitrides, oxides and carbides have been extensively studied in the last years because their unique physical and chemical properties [1-3]. The growing interest in producing thin films of these compounds arises due to the fact that many of them show novel properties as new electronic materials the including use as semiconductors, implants for quantum well and quantum dot devices, and in construction of solar cells and photovoltaic devices. The study of molecular clusters, which are herein reported, is an important feature of the production of thin films or of systems of finite size such as nanostructure materials with tailored [4] properties.

A variety of methods have been used to fabricate thin films and nanoparticles of these materials, including Molecular Beam Epitaxy, Radiofrequency sputtering and Ion sputtering, Spray pyrolysis, Chemical Vapor Deposition (CVD) and Pulsed Laser Ablation and Deposition (PLAD). In particular PLAD [5] has been shown to be a very powerful deposition method of all these materials. Compositional changes may occur during pulsed laser ablation and thin film deposition. A very important feature involved in thin film deposition by PLAD is the congruency of the vaporizing components compared to the solid and liquid films. The aim of various studies was to maintain the stoichiometry of the condensed phase. The loss of oxygen or nitrogen is very important for a balance of the processes. The addition of these gases is advisable in order their loss. Active oxygen or nitrogen could be more efficient with respect to the molecular elements [6]. A substrate of an atomic

\footnotetext{
†E-mail: aldo.mele@uniroma1.it
}

gas such as nitrogen or oxygen created by a radiofrequency (RF) discharge may establish a new equilibrium to contrast the loss. This same technique may be used to perform a chemical reaction between the atomic atmosphere and the ablated plume from a target made of single components such as metals or graphite [7].

\section{PRIMARY AND SECONDARY MECHANISMS}

Laser matter interaction. Laser ablation refers to a process of particle injection, named "primary mechanism" [8], similar to sputtering in which material removed rates typically exceed one tenth of monolayer per pulse. This is different in the characteristics with laser induced desorption which occur without any detectable mesoscopic surface modification and structure. The proportionality between yield and electron excitation density, underlines the partially yield, which is an "non-linear function" of the laser energy density (fluence) [9]. The expression of a "linear regime" applies in the second case, at a fluence below the threshold. Thus the most relevant feature between the two regimes is the change of the yield vs fluence. PLAD has also in most cases the unique capability in maintaining the stoichiometry of the thin film produced at high fluence.

A purely thermal model in the description of laser heating of the surface and subsequent material evaporation in the nanosecond and femtosecond regimes is inadequate as shown by the velocity distribution of the emitted particles which exceed by far the predicted values also at $5000 \mathrm{~K}$. Time resolved studies of laser desorption in metals performed using pulsed laser operating in the femtosecond regime have shown that for very fast processes, the metal may be considered as a 


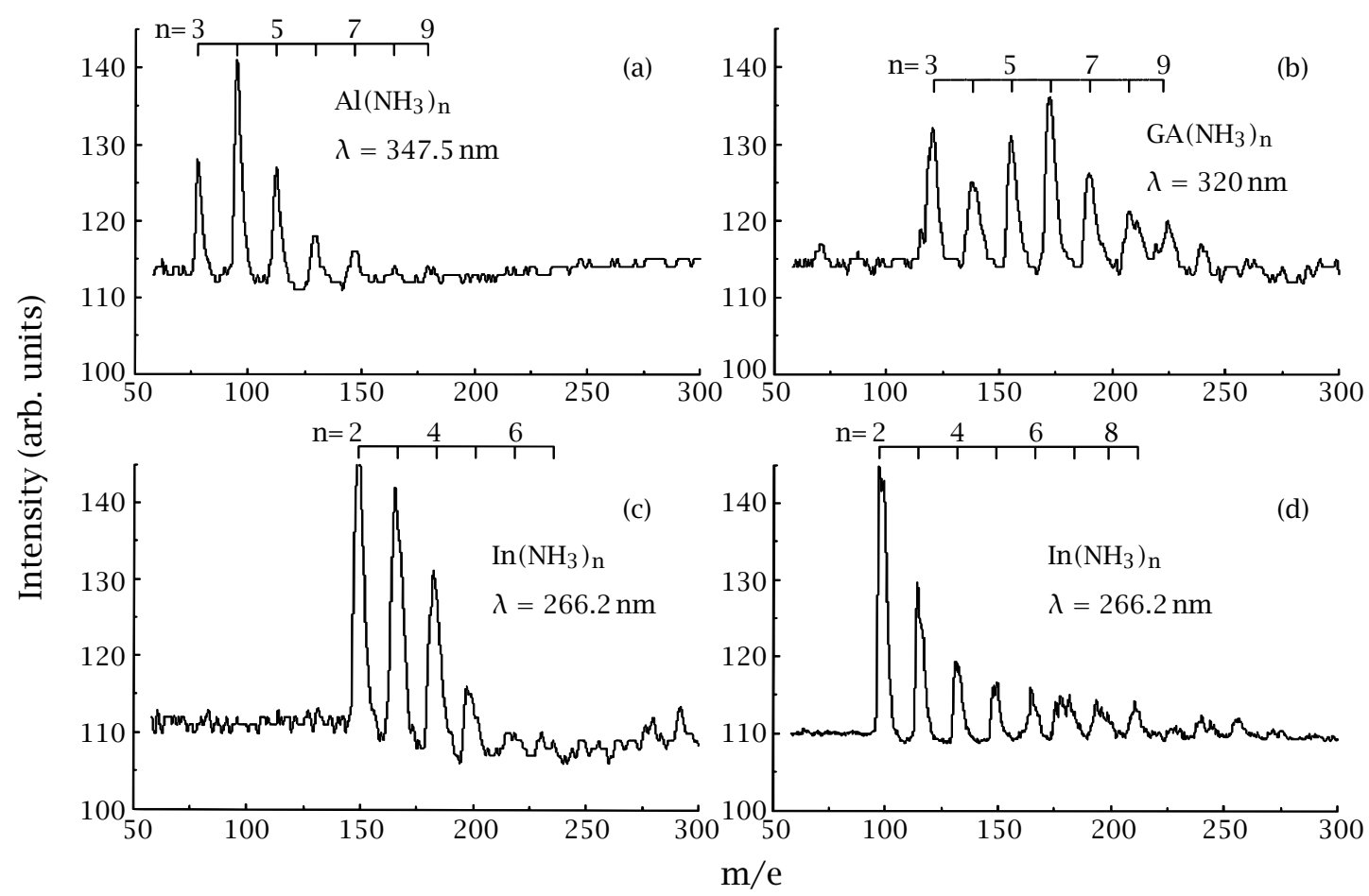

Figure 1. Set of mass spectra of $\mathrm{Al}\left(\mathrm{NH}_{3}\right)_{n}, \mathrm{Ga}\left(\mathrm{NH}_{3}\right)_{n}, \mathrm{In}\left(\mathrm{NH}_{3}\right)_{\mathrm{n}}$ and $\mathrm{Cu}\left(\mathrm{NH}_{3}\right)_{\mathrm{n}}$ clusters by reaction of $\mathrm{Al}, \mathrm{Ga}, \mathrm{In}$ and $\mathrm{Cu}$ plume in a stream of $\mathrm{NH}_{3}$ and $\mathrm{He}(10 \%)$. Stagnation pressure $4 \times 10^{5} \mathrm{~Pa}$. KrF eximer laser, wavelength $248 \mathrm{~nm}$ : Laser fluence $1 \mathrm{~J} / \mathrm{cm}^{2}$. Ionization wavelength: (a) $347.5 \mathrm{~nm}$, (b) $320 \mathrm{~nm}$, (c) $266 \mathrm{~nm}$ and (d) $290 \mathrm{~nm}$.

two temperature system of the electron and the lattice [10]. This behavior can be described by two coupled non linear partial differential equations to take in account the initial vaporization of the surface, and to evaluate the depth of ablation at the damage fluence threshold. Characterization of composition, and velocity distribution of the plume is performed through various techniques, such as Intensified Time Resolved Optical Imaging (ICCD), Optical Spectroscopy (Optical Multichannel Analyzer) and Mass spectrometry (Time of Fligth or Quadrupole Mass Spectrometers).

Laser ablation and clusters formation. At present there is a great interest in systems of finite and small size. Particularly interesting are systems with characteristics which may serve as the building blocks for nanostructures and clusters assembled materials. Small systems often displays unique properties which provide a challenge of considerable scientific interest [11]. The analysis of the gas phase cloud, moving away from the target, named "secondary mechanism" [8] is an important step in thin films fabrication. The questions to answer concern: chemical and structure identity, and chemical reactivity. Studies on laser ablation and clusters formation have been carried out by several techniques. The LAMMA apparatus has been first employed to analysis clusters by quadrupole mass spectrometry [12]. Clusters ions have been produced from several oxides of various elements. The use of this technique was pioneered to produce clusters of nitrides, oxides and carbides starting from targets of the bare elements. The formation of clusters precursor of thin film deposition was studied also by developing a more sophisticated laser ablation supersonic apparatus [13]. Reaction of ammonia with bare metal targets, such as Alluminum, Indium, Gallium and copper (Figure 1) have been examined in order to understand the mechanism of nitrides formation [1].

\section{EXPERIMENTAL METHODOLOGY}

Pulsed laser ablation. The laser ablation experiments were carried out in a multiport stainless steel vacuum chamber equipped with a gas inlet, a rotating multi-target and a heatable substrate holder [14]. The vacuum pressure of the deposition chamber was below 10-3 Pa whereas the reacting gas pressure during film depositions was $10 \mathrm{~Pa}$. The deposition temperature could be varied from room temperature up to $1000 \mathrm{~K}$. The fluence of the laser employed (Quantel Nd: YAG $581, \lambda=532 \mathrm{~nm}$, pulse duration $=7 \mathrm{~ns}$, repetition rate $=10 \mathrm{~Hz}$ ) has been kept nearly constant at $8 \mathrm{~J} / \mathrm{cm}^{2}$. The laser impinges on the target surface at an angle of $45^{\circ}$ with respect to the normal in a static gas atmosphere. All the targets were rotated at $2 \mathrm{rpm}$ during depositions. 


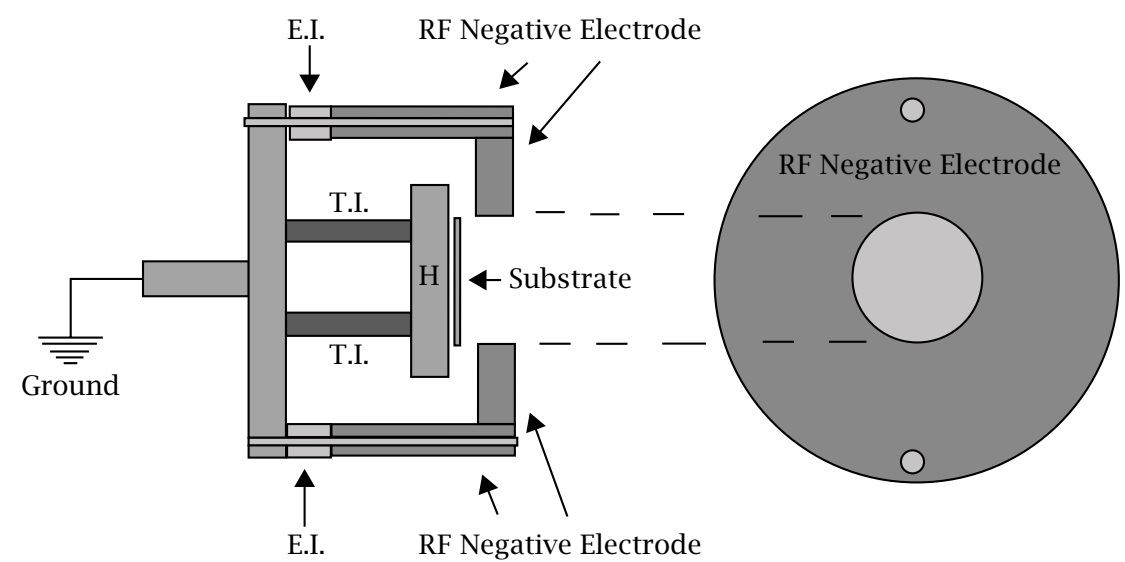

Cross Section

Front View

Figure 2. Schematic layout of the heatable sample holder (H) hooded by a stainless steel top-hat, with a $30 \mathrm{~mm}$ diameter hole, in which it is inserted the output of the matching network coming from the RF power generator. (E.I. = electrical insulator, T.I. = thermal insulator).

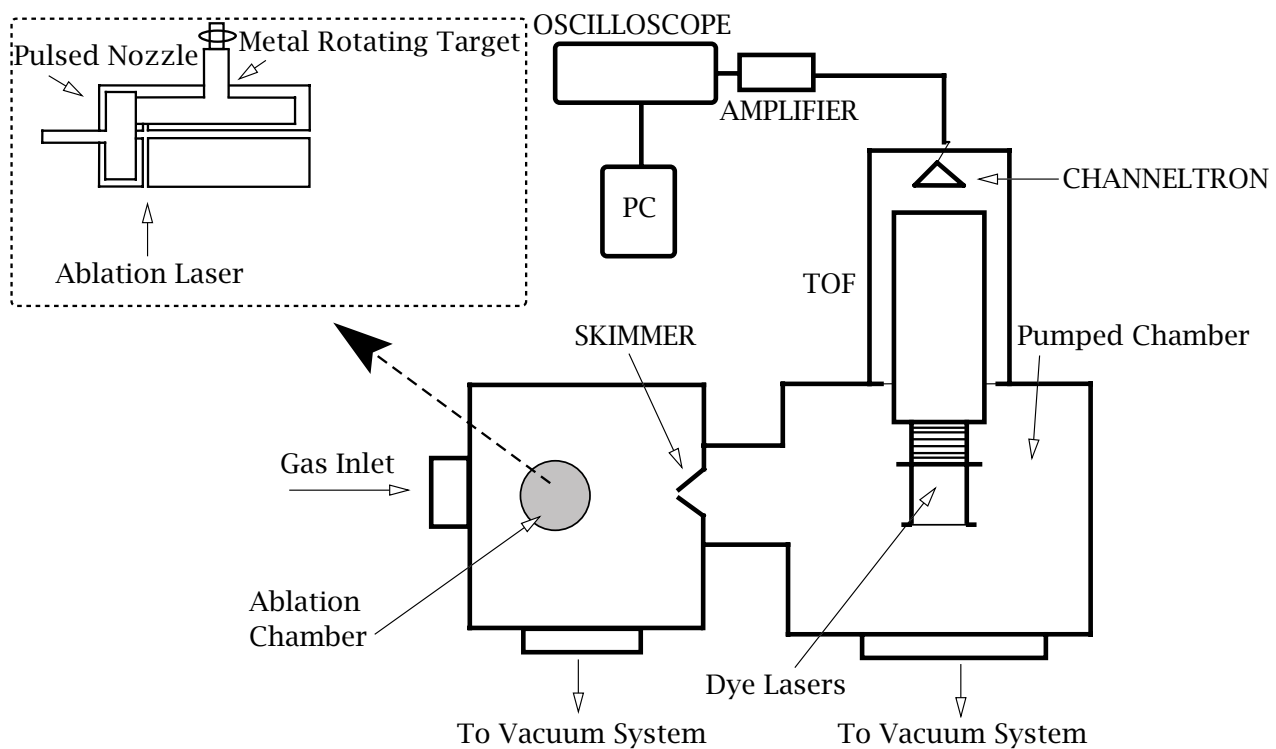

Figure 3. Schematic of the supersonic beam experimental apparatus employed for the production of clusters.

Radiofrequency assisted PLAD apparatus. The PLAD set up has been recently improved by employing a RF plasma system placed just above the substrate holder (Figure 2), maintaining the older electrically connected to the ground [15]. The substrate holder is surrounded by an isolated stainless steel top-hat connected to the RF generator, through a customized matching unit. A $3 \mathrm{~cm}$ diameter hole on the top-hat allows the deposition of the plume coming from the ablated target. The RF power generator is a $13.56 \mathrm{MHz}$ ENI Model OEM-6, maximum power output $650 \mathrm{~W}$.

Supersonic molecular beam apparatus. The experimental apparatus we have employed for the production of clusters is an instruments constructed in our laboratory and is reported in Figure 3 [13]. It consists of two vacuum chambers separated by a conical skimmer. In a small ablation chamber a mixture of reactive gases in He ranging from $0.1 \%$ to $100 \%$ at a stagnation pressure of (2-4) $10^{5} \mathrm{~Pa}$ is introduced through apulsed nozzle (200 s time duration and $1 \mathrm{~mm}$ diameter). In the same ablation chamber a rotating target is vaporized by a Nd : Yag laser doubled in frequency $(532 \mathrm{~nm}$, $0.15 \mathrm{~J} /$ pulse). The jet formed, after being skimmed, enters in a second chamber equipped with time of flight (TOF) mass spectrometer. The clusters in the beam are ionized by $\mathrm{Nd}$ : Yag pumped Dye laser or a KrF laser (= $248 \mathrm{~nm}, 0.5 \mathrm{~mJ} /$ pulse) and detected by a channeltron. The mass selected signals are recorded by digital oscilloscope, stored and processed on a PC. 


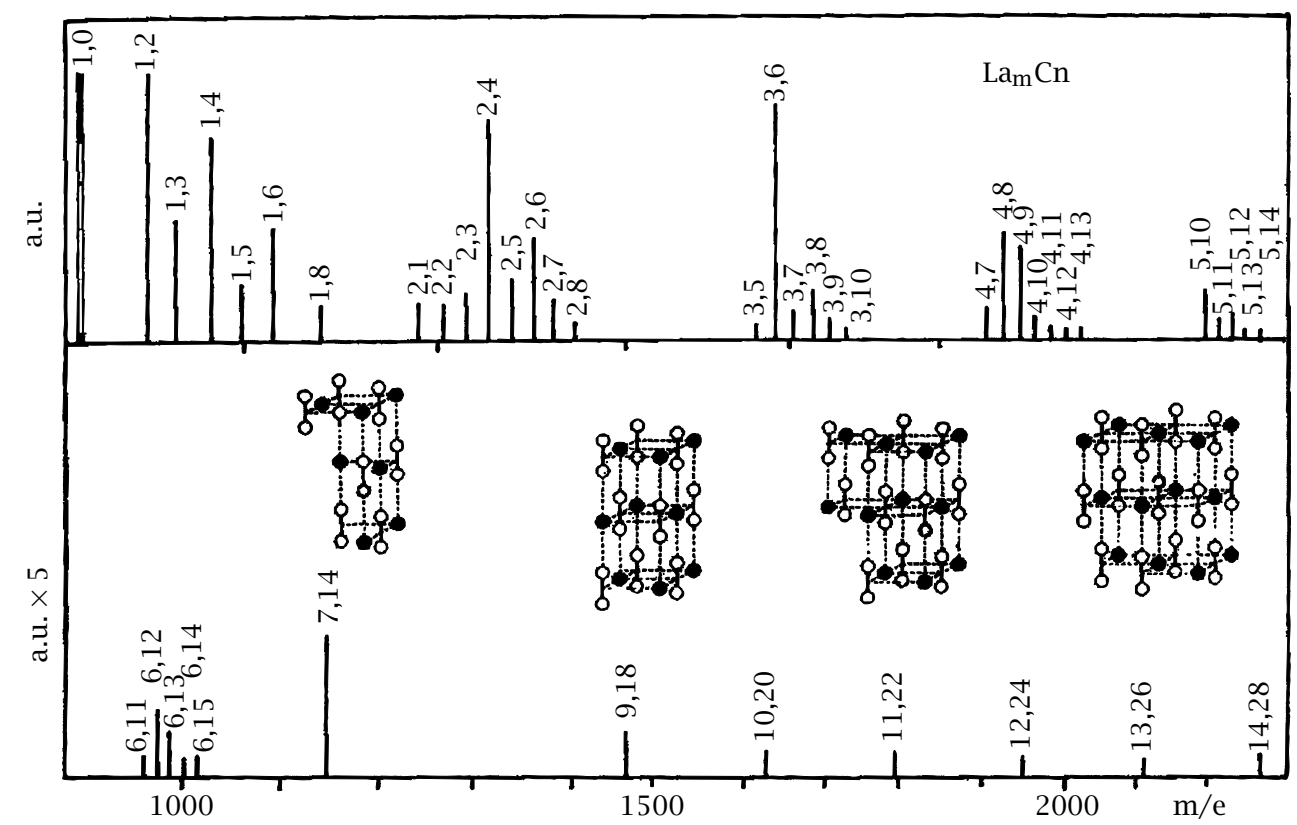

Figure 4. Typical LAMMA mass spectra of lanthanium carbide clusters $\mathrm{La}_{\mathrm{m}} \mathrm{C}_{\mathrm{n}}$ where $\mathrm{m}$ and $\mathrm{n}$ are (top) the index (bottom)of a few cluster structures $m=7$.

\section{RESULTS AND DISCUSSION}

Cluster production and analysis. Studies of cluster formation obtained by laser ablation of oxides have been analyzed by LAMMA spectrometry and have shown stoichiometry structure $\mathrm{M}_{\mathrm{n}} \mathrm{O}_{\mathrm{m}}^{+}$with chemical formula involving in integral values of $\mathrm{n}$ and $\mathrm{m}$ and non stoichiometry formula associated with lattice defect [12]. It has been found that the same oxide may be in various crystalline forms and present in different yield for low and high molecular weight in the mass spectra. More stable structures are usually formed according to their coordination number. However less stable sequence of clusters may also be found. Assuming a role of clusters in the formation of thin film, the growth of a stable structure is thus important data to be taken into account. The mass spectra of oxides indicate also the presence of fragmentation ions and particularly of oxygen negation ions. This fact would justify the addition of active oxygen to compensate this loss.

Other work on oxides concerns laser evaporation systems such as $\mathrm{Sc}_{2} \mathrm{O}_{3}, \mathrm{Y}_{2} \mathrm{O}_{3}$ and $\mathrm{La}_{2} \mathrm{O}_{3}$ (Figure 4) in a mixture with graphite, yielding ionized metal carbide of formula $\mathrm{Me}_{m} \mathrm{C}_{n}^{+}[16]$. The reaction takes place by a near complete reduction of the oxide by means of graphite. Two sequences of clusters have been observed in the mass spectra. The main sequence is $\left(\mathrm{MeC}_{2}\right) \mathrm{X}+$ together with carbide clusters of a more general formula $\mathrm{Me}_{\mathrm{m}} \mathrm{C}_{n}^{+}$ with a ratio of carbon to metal greater than 2 . This is probably due to the effect of carbon the atom density in the reaction region. This may find an interpretation in analogy with bare carbon .
Carbon clusters $C_{n}$ have been found to be formed from graphite by pulsed laser ablation up $\mathrm{n}$ larger than 60 , as analyzed by LAMMA apparatus [16]. The origin of the stability of neutral, positive and negative bare carbon cluster have been discussed and an even-odd effect which was found in the cluster stability has been explained in terms of molecular orbital model. Neutral and positively charged odd species have a (molecular orbital completely filled and are more stable than even species with only half filled $\pi$ orbitals.

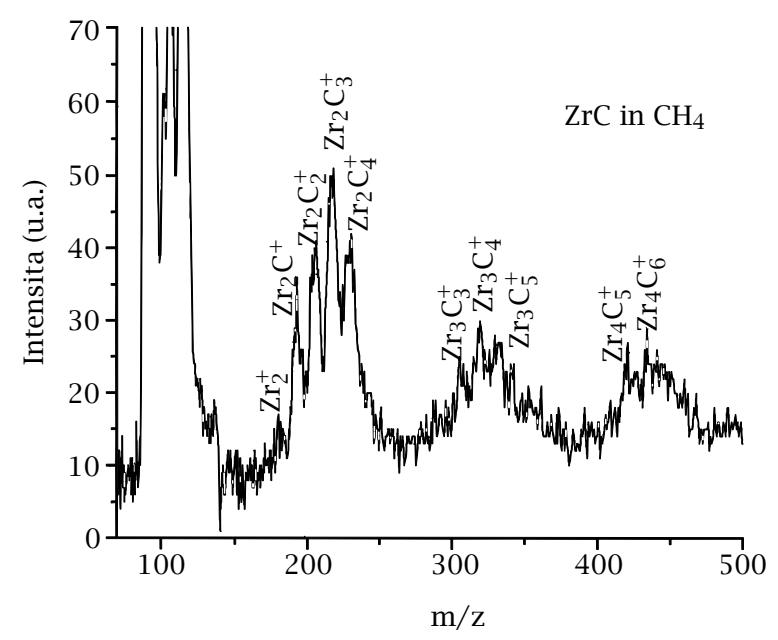

Figure 5. Non resonant one photon ionization TOF mass spectra of $\mathrm{Zr}_{\mathrm{n}} \mathrm{C}_{\mathrm{m}}$ clusters at $\mathrm{KrF}$ excimer laser ablation, wavelength $248 \mathrm{~nm}$ : Laser fluence $0.5 \mathrm{~mJ} /$ pulse. 
The photoionization mass spectra of zirconium carbide thin film of $\mathrm{Zr}_{\mathrm{m}} \mathrm{C}_{\mathrm{n}}$ obtained by ablation of a zirconium carbide target in the presence of methane is reported in Figure 5; the cluster growth of $n$ is up to 6 . The relative intensity depends on the concentration of methane in the beam. The studies of the cluster systems were chosen in order to ultimately determine the effect of the electron stability of the structure of the plasma components. The growth mechanism may be connected with the process of selective chemistry of the species leading to thin film formation and deposition.

Recent studies have discovered the formation of metals carbides, nitrides and oxides by a method based on tandem mass spectrometry [4]. These authors have discovered stable structures of metal carbides, nitrides and oxides $\mathrm{Me}_{8} \mathrm{C}_{1} 2$ which make a new class of molecular clusters termed Met-Cars. The stability of this species is based on a dodecahedron structure where the metal atom would occupied 8 unique positions.

Radiofrequency (RF) assisted thin film deposition. The present work is intended to show same results on PLAD thin film deposition from various materials and to established a relationship between the composition of the films with what is expected by "normal vaporization" of the target. The congruency or incongruency of the process is defined by comparing the two counterparts, films and targets, for the components changes.

A thermal treatment of the process considers that evaporation-decomposition is what really take place during the laser pulse. In the range of the laser irradiance of the experiments of about $10^{8} \mathrm{~W} / \mathrm{cm}^{2}$, it is assumed that the most removed from the target is due to vaporization. The incongruency of the balance in the process is due in the case of multicomponents target to the loss of one of the elements of the target. This is preferably the most volatile [6]. Other possible changes may take place in the gas phase, due to either the instability of the species or in the plasma which may decompose or to ion-molecule reactions which may take place between the species present in the plasma or induced by addition of ionized or neutral species to the vaporized materials. There is evidence that this may occur as shown by a comparison of the analysis of the plume and the composition of the thin film. The analysis of the plume by mass spectrometry or by optical methods have confirmed the view that the loss of component is quite large. Decomposition processes occur due to the thermal spike induced by laser surface interaction. Herein, we report the synthesis of boron-nitrogen and metal- oxygen materials by using atomic nitrogen and atomic oxygen created by a radiofrequency (RF) discharge [15, 17].

Boron nitrides thin films have been deposited on $\mathrm{Si}$ (100) substrate by reactive pulsed laser ablation of pure boron target in the presence of $\mathrm{N}_{2}$ buffer and $13.56 \mathrm{MHz}$ radiofrequency (RF) field apparatus shown in Figure 2.
The presence of the RF plasma allows the growth of the stable hexagonal boron (h BN) nitride from a boron target in a nitrogen gas atmosphere. The IR spectroscopy shows that RF plasma enhances the formation of hexagonal boron nitride (h BN) with respect to other phases obtained by non-assisted pulsed laser ablation.

Metal oxides thin films have been fabricated by the same apparatus. Target of tungsten trioxide and titanium oxide $\mathrm{TiO}_{2}$ have been irradiated [17] in the apparatus of Figure 2 by using $13.56 \mathrm{MHz}$ radiofrequency palsma, 10 Pa static atmosphere oxygen and a doubled frequency $\mathrm{Nd}$ : Yag laser $(\lambda=532 \mathrm{~nm})$. The deposition was performed on a heatable Si (100) substrate up to $1000 \mathrm{~K}$. A comparison between a conventional pulsed laser deposition system PLAD and the RF plasma assisted system displays the effect of the plasma. The addition thermal effect due to RF on the surface of the substrate may improve the properties of the film. It has been found that without the use of RF the adhesion is rather poor. The influence of the plasma on a better adhesion is confirmed by the adhesive tape tear test. Further the geometry of the deposit is more homogeneous and there is not preferential angle target and substrate, possibly due to a more distribution of the components of the plasma which reaches the substrate.

It is worth mentioning the results of zinc oxide thin film deposition by RF assisted ablation [18]. This oxide is a wide-band semiconductor, which exhibits a x-ray diffraction pattern of $\mathrm{ZnO}$ samples without and with a RF discharge. From a zinc target in the presence of an oxygen atmospheres the pattern shows ordered spectra by increasing the temperature. Beyond the effect of the temperature, which may produce films of different orientations, the (101) crystal growth orientation seems to be preferred if the film is produced by RF plasmaassisted PLAD.

\section{CONCLUSIONS}

The study reported in the present article shows that the analysis of the gaseous cloud formed above the target by pulsed laser ablation and moving towards the substrate may provide an insight of the components into the plasma which may be correlated with the thin film deposits. The cluster analysis is indicative of what are the changes of components occurring on the way to the target and their spatial and temporal distribution as measured by mass spectrometry. The experimental conditions may strongly affect the laser-target interactions and the following gas phase reactions in a short time $\left(\sim 10^{-5} \mathrm{~s}\right)$. The question is now what are the parameters which have the largest effect on the process. In the assumption of a thermal process, we look for the phase stability domains which are represented by $\mathrm{T}, \mathrm{P}, \mathrm{V}$ parameters and composition in gas phase diagrams. The laser power density and the radiofrequency atomic yield are both conditions which produce a large 
variability. The analysis of the plume components and the variability of the experimental set up of all systems may lead to a rather good inside of the phenomenological performance of the experiment, in order to establish the best trend. The few examples reported have tried to answer to basic questions of addressing the experiment to the right direction. Each system has to be examined separately in all variables, and a computational analysis approch may be useful to establish the correct trend.

\section{ACKNOWLEDGMENTS}

This work was partially supported by Progetto Strategico MSTA II of Consiglio Nazionale delle Ricerche (CNR) of Italy.

\section{REFERENCES}

[1] A. Mele, A. Giardini, T. Di Palma, C. Flamini, H. Okabe, and R. Teghil, Inter. Journ. of Photoenergy 3 (2001), 111.

[2] Y. Suda, H. Kawasaki, K. Doi, and S. Hiraishi, Thin Solid Films 374 (2000), 282; Ashok Kumar, H. L. Chan, and Rodica Alexandrescu, Advances in Science and Technology (Faenza, Italy) Surface Engineering 20 (1999), 131.

[3] R. Franchy, Surface Science Reports 38 (2000), 6; Stephen Muhl, Adriana Gaona-Couto, Juan Manuel Mendez, Sandra Rodil, Gonzalo Gonzalez, Alexander Merkulov, and Rene Asomoza, Hard Coatings based on Borides, Carbides \& Nitrides: Synthesis, Characterization \& Applications, Proceedings of the International Symposium, San Antonio, Febrauary 16-19, 1998, pp. 3-15.

[4] B. D. Leskiw, A. W. Castelman jr, Comptes Rendus Physique 3 (2002), 251.

[5] D. B. Chrisey and G. K. Hubler, Pulsed Laser Deposition of Thin Films, Wiley-Interscience Publication, New York, 1994.
[6] C. Flamini, A. Ciccioli, A. Giardini, and A. Mele, J. of Mat. Synthesis and Processing 93 (2001), 143.

[7] Chunming Niu, Yuan Z. Lu, and Charles M. Lieber, Science 261 (1993), 334.

[8] R. Kelly, A. Miotello, B. Braren, A. Gupta, and K. Casey, Nucl. Instr. and Meth. B 65 (1992), 187.

[9] R. F. Haglund jr, in Experimental Methods in the Physical Science, Laser Ablation and Deposition (J. C. Miller and R. F. Haglund j., eds.) Academic Press, Vol. 30, San Diego, 1998, p. 15.

[10] M. Satta, D. R. Ermer, M. R. Papantonakis, C. Flamini, R. F. Haglund jr, and A. Mele, Appl. Surf. Sci. 154-155 (2000), 172.

[11] Y. Xia, J. A. Rogers, K. E. Paul, and G. M. Whitesides, Chemical Reviews 99 (1999), 1823.

[12] A. Mele, D. Consalvo, D. Stranges, A. Giardini, and R. Teghil, Int. J. of Mass Spectrometry and Ion Process 95 (1990), 359.

[13] A. Giardini Guidoni, A. Mele, M. T. Di Palma, C. Flamini, S. Orlando, and R. Teghil, Thin Solid Films 295 (1997), 77.

[14] A. Mele, A. Giardini Guidoni, R. Kelly, C. Flamini, and S. Orlando, Appl. Surf. Sci. 109/110 (1997), 584.

[15] V. Marotta, S. Orlando, C. P. Parisi, and A. Santagata, Appl. Surf. Sci. 9656 (2002), 1.

[16] A. Giardini Guidoni, A. Mele, G. Pizzella, and R. Teghil, Z. Phys. D Atoms, Molecules and clusters 20 (1991), 89.

[17] A. Giardini, V. Marotta, A. Morone, S. Orlando, and C. P. Parisi, Appl. Surf. Sci. 197-198 (2002), 338.

[18] A. Giardini, V. Marotta, S. Orlando, A. Paladini, G. D’Amico, and G. P. Parisi, in Contributions to XIIIth Symposium on Atomic, Cluster and Surface Physics (SASP 2002), Going, Austria, February 1722, 2002.

[19] H. Cao, Y. Xu, D. Zhang, S.-H. Chang, S. T. Ho, E. W. Seeling, X. Liu, and R. P. H. Chang, Phys. Rew. Lett. 84 (2000), 5584. 


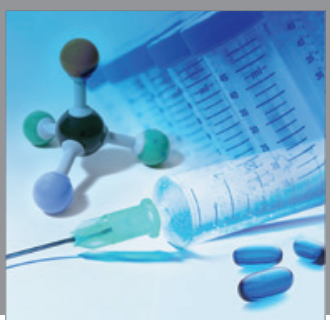

International Journal of

Medicinal Chemistry

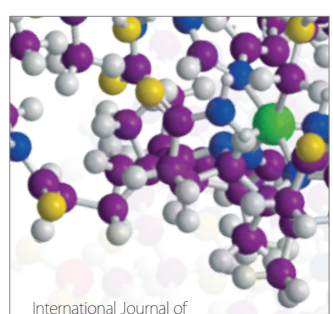

Carbohydrate Chemistry

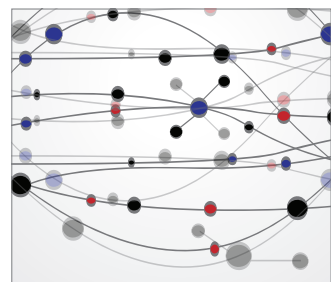

The Scientific World Journal
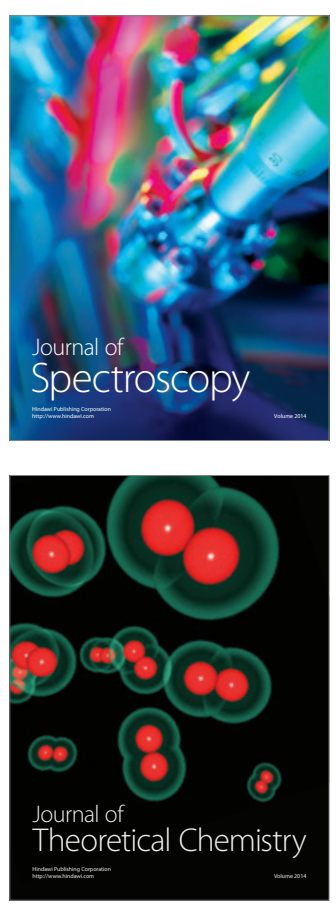
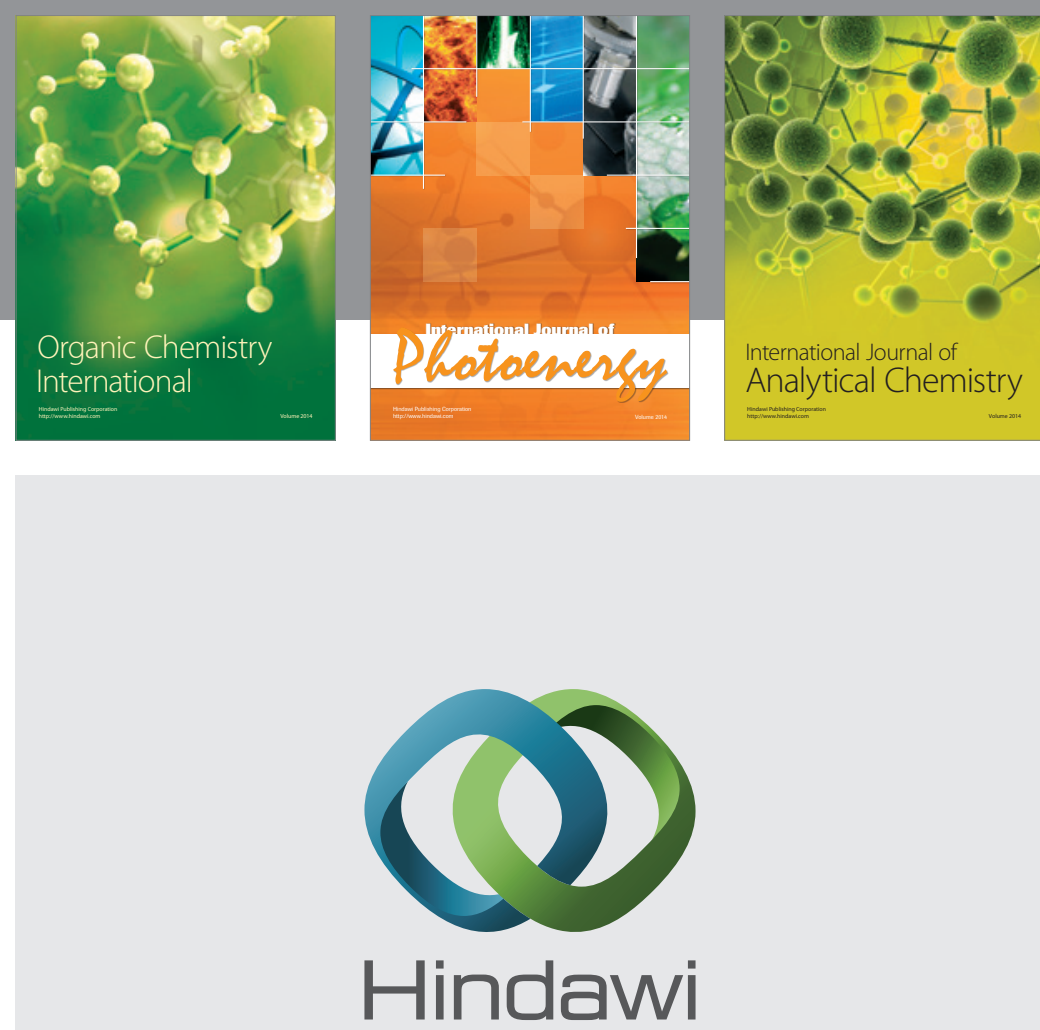

Submit your manuscripts at

http://www.hindawi.com
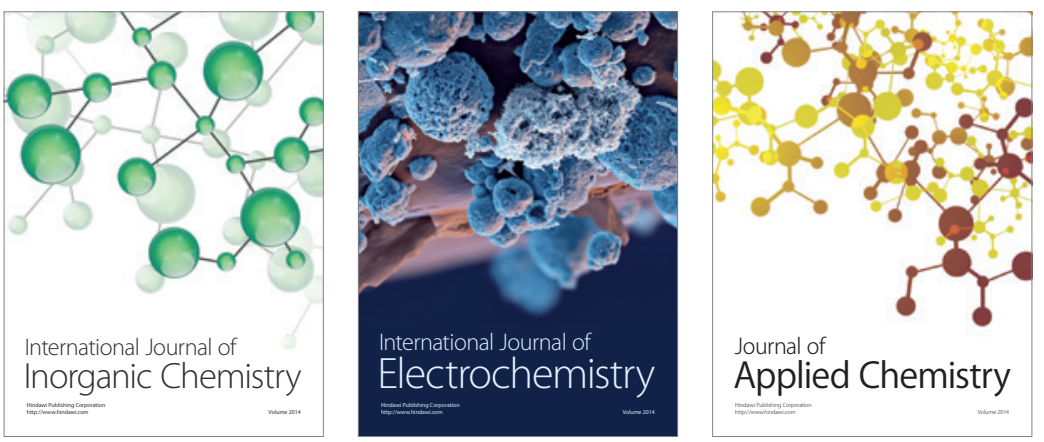

Journal of

Applied Chemistry
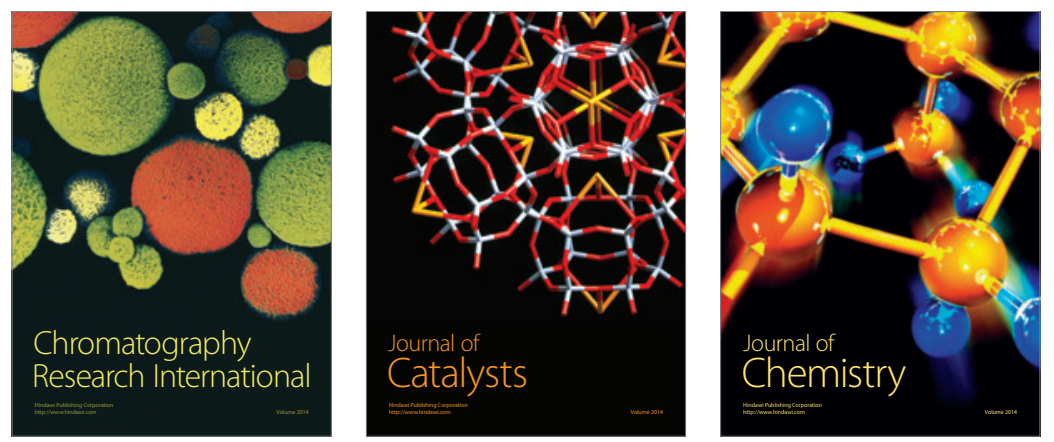
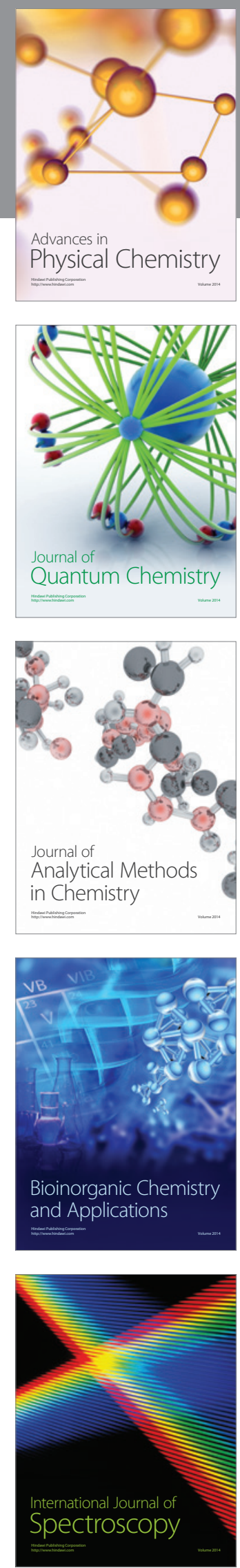